\title{
Ingenuity across field boundaries
}

\author{
Interdisciplinarity yields brilliant scientific innovations.
}

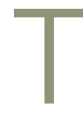

he ability to hypothesize - or, for that matter, to imagine - is probably unique to humans. By merging abstract thought with prior knowledge, we can rationally guess possible outcomes to a set of actions, find associations between apparently unrelated ideas or facts, and transfer ideas or thought processes from one field of knowledge to another. Human ingenuity has effectively no boundaries because it can cross fields of knowledge. Within the ambit of health, many of the great discoveries and inventions arose at the interface of medicine with physics, chemistry, or molecular and cell biology. Great examples abound, from the wellknown accidental discoveries of penicillin, $\mathrm{X}$-rays and warfarin, to the inventions that led to the polio vaccine, general anaesthesia and magnetic resonance imaging.

As a naturally interdisciplinary field, biomedical engineering has contributed to the development of a number of innovations. A few of the most notorious examples are computer tomography, cochlear implants, pacemakers, artificial joints, deep-brain stimulation, vascular stents, artificial skin and, more recently, cell-reprogramming methods, robotic surgery and 'omics' tools. These and many other biomedical or engineering innovations have changed or are changing healthcare, and have been instrumental in advancing our knowledge of the biology of the human body and the mechanisms by which diseases alter it. As both a biomedical journal and an engineering journal, Nature Biomedical Engineering disseminates technological innovations and the advantageous application or optimization of existing innovations - that significantly drive forward the understanding of human physiology and disease, the application of diagnosis or therapy, the monitoring or improvement of health, or the delivery of healthcare interventions. In this respect, this issue highlights a range of innovations that exemplify the fruits of human ingenuity at work.

Azita Emami, Mikhail Shapiro and colleagues describe a new concept for the precise and non-invasive localization of microdevices implanted in the body. The researchers took inspiration from the principles of nuclear magnetic resonance, in particular that nuclear spins resonate at a frequency that depends on the externally applied magnetic field, to design integrated circuits that emit radiofrequency signals at a magnetic-field-dependent frequency (shifted from the excitation frequency generated by a previously applied radiofrequency pulse), which can then be used to determine the position of the circuit in a magnetic-field gradient. The researchers tested the concept in devices implanted subcutaneously in anaesthetized mice. In the future, these devices could be incorporated into implantable wireless sensors and swallowable drug-release pills to transmit their location and information about their state.

Also at the juncture of physics and biomedicine, Xudong Fan and colleagues report on a microscope that detects cancer cells in tissue biopsies stained with fluorescent nucleic acid markers on the basis of the cells' lasing output. To make cells emit laser light, the tissue is sandwiched between two closely spaced mirrors that are reflective at the emission wavelength for the staining fluorophore (yet transparent to the excitation light). Owing to the lower lasing threshold of cancerous cells, presumably because of their larger nucleic acid content, the microscope is highly sensitive to them and may prove to be advantageous in the detection of cancer during its early stages.

Matthew Dalby and collaborators demonstrate that a bioreactor applying highfrequency, nanometric vibrations to human bone-marrow stromal cells cultured in a collagen gel induces their differentiation into osteoblasts, which mineralize the gel matrix, and that the force is partly transduced from the bioreactor to the cells by mechanosensitive cation-channel proteins in the cells' lipid membrane. To measure the amplitude of the vibrations, the researchers used a laser-interferometry method akin to those developed for the detection of gravitational waves. The bioreactor could eventually be used to make mineralized collagen for bone regeneration.

By exploiting click chemistry, Youqing Shen and co-authors synthesized a simple dendrimer that, when intravenously injected into mice, inhibits tumour growth and metastasis, with efficacies higher than those of some anticancer drugs and without causing observable toxicity. The researchers

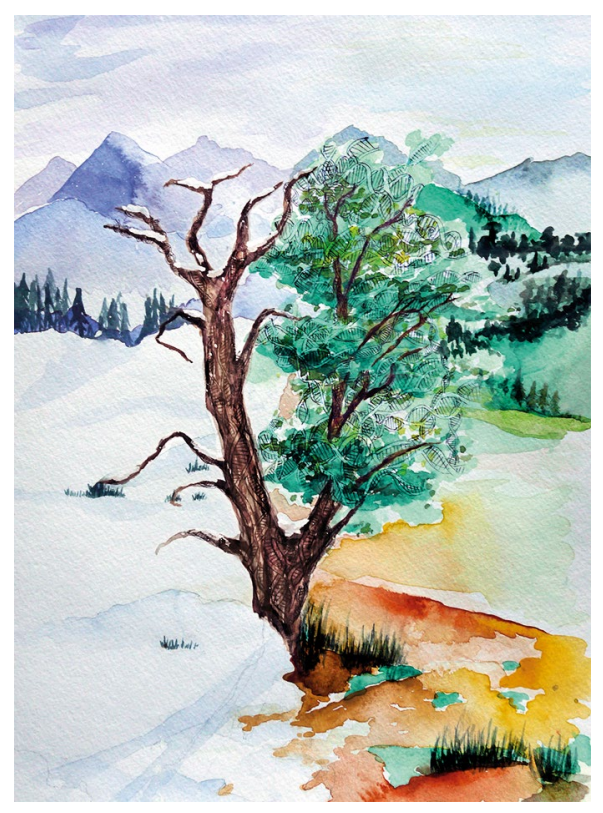

Field boundaries. Credit: Cindy Thaung

show that the potent anticancer activity results from the ability of the dendrimer to deplete bioavailable copper, a critical element for tumorigenesis.

And David Yu Zhang and collaborators combined knowledge of thermodynamics and molecular biology to design a polymerase-chain-reaction method based on a competitive hybridization reaction that selectively enriches hundreds of potential rare DNA-sequence variants simultaneously, and that is highly robust and efficient within a wide annealing-temperature range $\left(56-64^{\circ} \mathrm{C}\right)$. The method is inexpensive and fast, and could therefore be advantageously applied to monitor cancer recurrence from a patient's cell-free DNA.

In a Comment, Lena Maier-Hein and colleagues argue that the operating room needs to take advantage of large-scale data and machine learning to assist the surgical team and vastly improve patient outcomes. Irrespective of developments in artificial intelligence, however, ingenuity will remain uniquely human for the foreseeable future. $\square$

Published online: 12 September 2017 DOI: 10.1038/s41551-017-0138-1 